\title{
Österreichische Bewegungsempfehlungen für Frauen während der Schwangerschaft und danach, für Kinder im Kindergartenalter und für Kinder und Jugendliche
}

\author{
Austrian Physical Activity Recommendations for Women during \\ and after Pregnancy, Children of Kindergarten age, Children, and \\ Adolescents
}

\section{다 (i) $(\mathrm{B})($}

\section{Autoren}

Susanne Ring-Dimitriou ${ }^{1}$, Karin Windsperger ${ }^{2}$, Rosemarie Felder-Puig ${ }^{3}$, Beate Kayer ${ }^{4}$, Verena Zeuschner ${ }^{5}$, Piero Lercher ${ }^{6}$

Institute

1 Interfakultärer Fachbereich Sport- und Bewegungswissenschaft, Universität Salzburg, Salzburg, Austria

2 Universitätsklinik für Frauenheilkunde, Medizinische Universität Wien, Wien, Austria

3 Fachbereich Kinder- und Jugendgesundheit, Institut für Gesundheitsförderung und Prävention (IfGP), Wien, Austria

4 Fachbereich Hebammen, Fachhochschule Campus Wien, Wien, Austria

5 Geschäftsbereich Fonds Gesundes Österreich, Gesundheit Österreich GmbH, Wien, Austria

6 Teaching Center, Medizinische Universität Wien, Wien, Austria

\section{Schlüsselwörter}

gesundheitswirksame Bewegung, Kindheit, Jugend, Gravidität, Richtlinien

\section{Key words}

health-enhancing physical activity, childhood, youth, pregnancy, guidelines

\section{Bibliografie}

Gesundheitswesen 2020; 82 (Suppl. 3): S177-S183

DOI 10.1055/a-1191-4060

ISSN 0949-7013

(C) 2020. The Author(s).

This is an open access article published by Thieme under the terms of the Creative Commons Attribution-NonDerivative-NonCommercial-License, permitting copying and reproduction so long as the original work is given appropriate credit. Contents may not be used for commercial purpose, or adapted, remixed, transformed or built upon. (https://creativecommons. org/licenses/by-nc-nd/4.0/)

\author{
Korrespondenzadresse \\ Prof. Susanne Ring-Dimitriou \\ Interfakulrärer Fachbereich Sport- und \\ Bewegungswissenschaft \\ Universität Salzburg \\ Schlossallee 49 \\ 5400 Hallein \\ Austria \\ susanne.ring@sbg.ac.at
}

\section{ZUSAMMENFASSUNG}

Basierend auf bereits dargelegten internationalen Empfehlungen werden in diesem Beitrag die Österreichischen Bewegungsempfehlungen für Frauen während der Schwangerschaft und danach, für Kinder im Kindergartenalter sowie für Kinder und Jugendliche in diesem Beitrag vorgestellt. Für Frauen in der Schwangerschaft und in den ersten Monaten nach der Entbindung weichen die Bewegungsempfehlungen nicht von jenen der Erwachsenen ab. Lediglich bei vorliegenden gesundheitlichen Problemen, kann es zu Bewegungseinschränkungen kommen. Kindern unter 6 Jahren sollten so oft wie möglich Bewegungsgelegenheiten geboten werden und sich bis zu 3 Stunden täglich mit mittlerer und hoher Intensität auf vielfältige Weise bewegen können, um ein gesundes Aufwachsen zu ermöglichen. In der Altersgruppe der 6 bis 18-Jährigen wird tägliche Bewegung im Umfang von mindestens einer Stunde empfohlen. Bei der Auswahl der Bewegungsformen sollte der Entwicklungsstand und das Aktivitätsausmaß von Kindern und Jugendlichen beachtet werden, um ein freudvolles überdauerndes Bewegungsverhalten zu garantieren. Die Bewegungsempfehlungen dienen der Verbreitung im Bereich der Gesundheitsförderung und stellen eine Grundlage für die Entwicklung und Evaluation von gesundheitswirksamen Bewegungsprogrammen dar.

\section{ABSTRACT}

In this article, based on international recommendations, we present the Austrian physical activity recommendations for 
women during and after pregnancy, children of kindergarten age, children, and adolescents. For women during pregnancy and in the first months after delivery, the exercise recommendations do not differ from those for adults. Movement restrictions apply only if health problems are present. Children under 6 years of age should engage in a variety of motor tasks for up to 3 hours a day at medium and high intensity levels, so that they can grow up healthy. In the age group of 6 to 18 years of age, daily exercise of at least one-hour duration is recommended. When choosing the type of exercise, the level of development and the activity levels of children and adolescents should be taken into account. The exercise recommendations are intended for dissemination in the field of health promotion and to serve as the basis for the development and evaluation of health-promoting exercise programs in Austria.

\section{Einleitung}

In diesem Beitrag werden die Österreichischen Bewegungsempfehlungen für die Zielgruppen ,Frauen während der Schwangerschaft und danach“, ,Kinder im Kindergartenalter' sowie ,Kinder und Jugendliche“ vorgestellt [1].

Die Ausführungen sind das Ergebnis eines intensiven, konsensualen Prozesses (2018-2020) mit Expertinnen und Experten der jeweiligen Fachgebiete sowie in Kooperation mit dem Auftraggeber, dem Fonds Gesundes Österreich, ein Geschäftsbereich der Gesundheit Österreich GmbH.

Unser Ziel war es, aktuelle internationale und nationale Empfehlungen in den österreichischen Kontext der Gesundheitsförderung einzuordnen und daraus Richtlinien für Akteure und Akteurinnen, die in unterschiedlichen Funktionen in der Public Health in Österreich tätig sind, abzuleiten. Dabei diente uns die aktuelle und umfangreiche Literaturarbeit des Physical Activity Guidelines Advisory Committee aus den USA als Vorlage [2]. Die in diesem Beitrag verwendeten trainingswissenschaftlichen Begriffe können im Detail bei Miko et al. in diesem Sonderheft nachgelesen werden und werden hier nicht weiter erörtert [3].

\section{Bewegung und Sport für Frauen während der Schwangerschaft und danach}

Regelmäßige körperliche Aktivität und Sport fördern die Gesundheit generell und haben für Schwangere grundsätzlich dieselben gesundheitlichen Vorteile wie für alle anderen Erwachsenen. Daher sollen alle gesunden Frauen mit einer unkomplizierten Schwangerschaft zu regelmäßiger Bewegung mit mittlerer Intensität als Teil eines gesunden Lebensstils motiviert werden.

Gesunde Frauen, die während der Schwangerschaft regelmäßig Bewegung ausüben, verbessern oder erhalten unter anderem die Leistungsfähigkeit ihres Herz-Kreislauf-Systems, reduzieren das Risiko einer übermäßigen Gewichtszunahme sowie das Risiko für Schwangerschaftshochdruck, Schwangerschaftsdiabetes, Präeklampsie und Geburtskomplikationen. Nach der Geburt zeigen sich positive Effekte wie eine kürzere benötigte Erholungszeit, ein besseres psychisches Wohlbefinden (Reduktion von depressiven Verstimmungen und Stimmungsschwankungen) sowie eine Stärkung des Beckenbodens und damit die Vorbeugung einer späteren Inkontinenz [4, 5].

Wissenschaftliche Studien zeigen auch, dass regelmäßig ausdauerorientierte Bewegung mit mittlerer Intensität während der Schwangerschaft weder mit einem erhöhten Risiko Kinder mit niedrigem Geburtsgewicht zur Welt zu bringen, noch eine Früh- oder Fehlgeburt zu erleiden, verbunden ist. Körperlich aktive Schwangere müssen also grundsätzlich keine negativen Auswirkungen auf ihr Kind befürchten und profitieren hinsichtlich des Gesundheitsnutzens selber stark davon [5-8].

Nach der Geburt hat körperliche Aktivität mit mittlerer Intensität keine negativen Auswirkungen auf die Menge und Zusammensetzung der Muttermilch sowie auf das Wachstum des Säuglings [4], bringt aber für die Mutter die beschriebenen positiven Effekte.

\section{Zielgruppe}

Die Bewegungsempfehlungen gelten sowohl für gesunde Frauen während einer unkomplizierten Schwangerschaft als auch für Frauen nach einer Spontangeburt, unabhängig davon, ob sie vor der Schwangerschaft regelmäßig körperlich aktiv waren oder nicht.

\section{Empfehlungen für Frauen während einer problemlosen Schwangerschaft}

Frauen, die bis zur Schwangerschaft nicht regelmäßig körperlich aktiv waren, sollen jede Gelegenheit nützen, körperlich aktiv zu werden. Vor allem der Wechsel von „körperlich inaktiv“ zu „ein wenig körperlich aktiv“ ist ein wichtiger erster Schritt. Zur Entwicklung, Erhaltung und Durchblutung der Muskulatur werden tägliches Beckenbodentraining und zusätzlich muskelkräftigende Übungen ohne Pressatmung an 2 oder mehr Tagen der Woche empfohlen.

Um die Gesundheit zu fördern und zu erhalten, werden mindestens 150 Minuten ( 2 1 12 Stunden) pro Woche ausdauerorientierte Bewegung mit mittlerer Intensität empfohlen. Bereits vor der Schwangerschaft aktive Frauen, die diese Empfehlungen überschreiten, können die gewohnten Bewegungs- und Sportaktivitäten im bisherigen Umfang weiterführen, solange sie sich dabei wohlfühlen. Gegebenenfalls können Art und Technik angepasst sowie Dauer oder Intensität reduziert werden. Langandauerndes Sitzen soll vermieden beziehungsweise immer wieder durch Bewegung unterbrochen werden. 


\section{Empfehlungen für gesunde Frauen nach einer Spontangeburt}

Gezieltes, angeleitetes Beckenbodentraining soll von allen Frauen gleich nach der Geburt begonnen und bis zu 6 Monate fortgesetzt werden. Ab 4 bis 6 Wochen nach der Entbindung wird empfohlen, den Bewegungsumfang schrittweise zu steigern bis die Bewegungsempfehlungen für Erwachsene wieder erreicht werden, nämlich:

- An 2 oder mehr Tagen der Woche muskelkräftigende Übungen durchführen, bei denen alle großen Muskelgruppen berücksichtigt werden.

- Mindestens 150 Minuten (2 1 1 2 Stunden) pro Woche ausdauerorientierte Bewegung mit mittlerer Intensität ausüben.

- Langandauerndes Sitzen soll vermieden beziehungsweise immer wieder durch Bewegung unterbrochen werden.

\section{Erläuterungen}

\section{Aktivitätsstatus}

Für Frauen, die bis zur Schwangerschaft nicht regelmäßig körperlich aktiv waren, ist jeder Schritt hin zu mehr Bewegung wichtig, da dadurch die Gesundheit von Mutter und Kind gefördert wird. Es wird empfohlen, den Bewegungsumfang pro Woche langsam zu steigern. Zuerst geht es darum, möglichst täglich Bewegungseinheiten in den Alltag einzubauen. Anschließend kann die Dauer der Bewegungseinheiten erhöht werden bis die 150 Minuten Bewegung mit mittlerer Intensität pro Woche erreicht sind.

Frauen, die bereits vor der Schwangerschaft körperlich aktiv waren, können die gewohnten Bewegungs- und Sportaktivitäten im bisherigen Umfang weiterführen. Gegebenenfalls soll die sportliche Aktivität im 2. und 3. Trimester reduziert werden [8].

Leistungssportlerinnen, die auch während der Schwangerschaft ihr Training fortsetzen wollen, benötigen besondere medizinische Aufsicht und sind im interdisziplinären Setting, z. B. durch Trainer/ innen und Sportärztinnen/-ärzte, zu betreuen.

\section{Art der Bewegung}

Bei der Auswahl gesundheitswirksamer Bewegung soll die Sicherheit für Mutter und Kind oberste Priorität haben. Zum Erreichen

- Tab. 1 Geeignete Bewegungsformen mit mittlerer Intensität während einer problemlosen Schwangerschaft.

\begin{tabular}{|l|}
\hline Bewegungsformen \\
\hline Zügiges Gehen oder Nordic Walking \\
\hline Wandern (bis 2000m Seehöhe) \\
\hline Fahren am Fahrradergometer \\
\hline $\begin{array}{l}\text { Schwangerschaftsgymnastik (z. B. schwangerschaftsadaptiertes Yoga } \\
\text { oder Pilates) }\end{array}$ \\
\hline Low-impact Aerobic \\
\hline Schwimmen oder Wassergymnastik \\
\hline Tanzen \\
\hline
\end{tabular}

der Bewegungsempfehlungen können Bewegungsminuten mit mittlerer Intensität zusammengezählt werden. Dabei werden die in $>$ Tab. 1 dargestellten ausdauerorientierten Bewegungsformen als geeignet angesehen.

Sportarten mit erhöhtem Verletzungsrisiko und/oder hohen Belastungsintensitäten wie Fußball, Tennis, Squash, Reiten, Alpinskifahren, Gewichtheben oder körperliche Aktivitäten über 2000 Meter Seehöhe sollen vermieden werden.

Ergänzend wird tägliches Beckenbodentraining während der Schwangerschaft und besonders nach der Geburt empfohlen, um die Festigkeit und Durchblutung dieser Muskulatur zu fördern und späterer Inkontinenz vorzubeugen (Kahlmeier et al. 2018). Hervorzuheben ist, dass Beckenbodentraining unter Anleitung zu einer Erhöhung der Trainingswirksamkeit führen kann [4, 5, 8, 9]. Besonderes Augenmerk sollte nach der Geburt auch auf Übungen gelegt werden, die der Rückbildung der Rektusdiastase (= Auseinanderstehen der geraden Bauchmuskeln) dienen. Die Rektusdiastase entsteht physiologisch ca. ab dem 5. Schwangerschaftsmonat.

\section{Wöchentlicher Bewegungsumfang}

Der wöchentliche Bewegungsumfang setzt sich aus der Häufigkeit, der Intensität und der Dauer der Bewegungseinheit zusammen.

Idealerweise soll die körperliche Aktivität auf mehrere Tage in der Woche, aber zumindest auf nicht weniger als 3 Tage pro Woche verteilt werden. Günstiger ist es, jeden Tag körperlich aktiv zu sein. Die Dauer der Bewegungseinheit hängt vom Fitnessniveau ab. Auf jeden Fall ist es wichtig, ausreichend zu trinken, weil während der Schwangerschaft erhöhter Flüssigkeitsbedarf besteht [4]. Ab etwa der 16. Schwangerschaftswoche soll außerdem Sport im Liegen vermieden werden [4,5].

Besonders wichtig während der Schwangerschaft ist die Wohlfühlkomponente. Das bedeutet, dass Bewegungs- und Sportaktivitäten beim Auftreten von Beschwerden oder Unwohlsein abgebrochen werden sollen. Das Motto lautet hier „Hören Sie auf Ihren Körper und treffen Sie die richtigen Anpassungen “ $[5,8]$.

Auch während der Schwangerschaft ist das subjektive Belastungsempfinden ein besserer Ratgeber bezüglich der Steuerung der Belastungsintensität, als bspw. eine Herzfrequenzmessung oder andere gerätebasierte Rückmeldesysteme der Belastungsintensität.

Nach der Geburt: Generell gilt es, ab 4 bis 6 Wochen nach der Geburt den ausdauerorientierten Bewegungsumfang langsam zu steigern. Für den Wiedereinstieg werden beckenbodenschonende Sportarten (z. B. Fahrradfahren, Nordic Walking, Schwimmen) empfohlen. Bei Sportarten, durch die Sehnen, Bänder und Muskulatur stark belastet werden, ist frühestens nach 12 Wochen wieder ein volles Training zu empfehlen. Die Expertinnen und Experten weisen darauf hin, dass High-Impact-Belastungen (z. B. Joggen, Springen) erst wieder 4 bis 6 Monate nach der Geburt ausgeübt werden sollen. Abhängig vom Geburtsverlauf bzw. vom Trainingszustand kurz vor der Geburt können diese Wartezeiten auch variieren, weshalb das Sporttreiben individuell anzupassen ist.

\section{Besondere Hinweise}

\section{Stillen und Bewegung}

Generell haben Bewegung und Sport mit mittlerer Intensität keine Auswirkungen auf das Stillen. Wichtig ist, dass stillende Mütter auf eine ausreichende Flüssigkeits- und Nährstoffzufuhr achten. Stillen oder Abpumpen vor der Bewegung kann in Betracht gezogen wer- 
den, um das unangenehme Gefühl spannender Brüste zu vermeiden. Ein besonders stützender BH oder ein Sport-BH können deutlich zum Wohlbefinden beitragen.

\section{Bewegung nach einem Kaiserschnitt}

Es wurden keine Bewegungsempfehlungen für Frauen nach einem Kaiserschnitt, der in Österreich bei rund $30 \%$ der Geburten durchgeführt wird [10], formuliert, weil individuell abzuklären ist, wann aus medizinischer Sicht mit einer schrittweisen Steigerung des Bewegungsumfanges begonnen werden kann. Hierzu eignet sich die fachärztliche Kontrolluntersuchung 6 bis 8 Wochen nach der Entbindung. Als Ausnahme ist hier das Beckenbodentraining anzusehen, mit welchem je nach individueller Situation, vorzugsweise angeleitet, schon früher begonnen werden soll.

\section{Schwangere mit gesundheitlichen Problemen und/oder Kompli- kationen in der Schwangerschaft}

Bei Frauen mit gesundheitlichen Problemen und/oder Komplikationen in der Schwangerschaft kann Bewegung vielfältige positive Effekte haben, kann aber auch kontraindiziert sein [5]. Bei Vorliegen der folgenden Pathologien soll diesbezüglich eine fachärztliche Begutachtung stattfinden $[4,5,8]$ :

a. Relative Kontraindikationen

- wiederholte Fehlgeburten

- Schwangerschaftsbluthochdruck

- Frühgeburt in einer vorangegangenen Schwangerschaft

- kardiovaskuläre und/oder respiratorische Erkrankungen

- Anämie

- Unterernährung

- Essstörungen

- Zwillingsschwangerschaft ab der 28. Schwangerschaftswoche

b. Absolute Kontraindikationen

- vorzeitiger Blasensprung

- vorzeitige Wehen

- erhöhtes Frühgeburtsrisiko

- anhaltende vaginale Blutung vor allem im 2. und 3. Trimester

- Placenta praevia nach der 28. Schwangerschaftswoche

- Präeklampsie

- Zervixinsuffizienz oder liegende Cerclage (operative Umschlingung des Gebärmutterhalses mit einem Faden)

- Wachstumsstörung beim Fötus

- höhergradige Mehrlingsschwangerschaft

- nicht bzw. schlecht eingestellter Diabetes mellitus Typ 1

- nicht bzw. schlecht eingestellter Bluthochdruck

- nicht bzw. schlecht eingestellte Schilddrüsenerkrankungen

- andere bedeutende medizinische Bedingungen

\section{Bewegungsempfehlungen für Kinder und Jugendliche ohne und mit Körper-, Sinnes- oder Mentalbehinderung}

Die Kindheits- und Jugendjahre sind wichtige Jahre für die Entwicklung eines Menschen, unter anderem auch in Bezug auf die Bewegungskompetenz und das Gesundheitsverhalten sowie für die Entwicklung körperlicher und geistiger Fähigkeiten [3].
Bereits für Kinder unter 6 Jahren wurden bedeutsame gesundheitsfördernde Effekte bei einem Bewegungsumfang von mindestens einer Stunde täglich für die kognitive und psychische Entwicklung sowie für die Entwicklung der Knochenstruktur und das Körpergewicht festgestellt [2,11-15].

Für Kinder und Jugendliche im Alter von 6 bis 18 Jahren ist die Beweislage umfangreicher. Im Vergleich zu wenig körperlich aktiven Kindern verfügen gesundheitswirksam aktive Kinder über eine höhere Fitness und weisen signifikant bessere Entwicklungseffekte in Bezug auf den Bewegungsapparat, das Körpergewicht, das Kreislaufsystem, den Stoffwechsel, die psychische Gesundheit und die schulische Leistung auf [2].

Vor dem Hintergrund eines gesundheitswirksamen Lebensstils wird die Rolle der körperlichen Aktivität gerade um eine wichtige Facette erweitert. Ergänzend zum Energiebilanzmodell wird Bewegung nicht nur als energieverbrauchende „Output-Komponente“ betrachtet, sondern als Vermittlerin der Regulation des Essverhaltens und der Verstoffwechselung von Nährstoffen [16]. In diesem Zusammenhang konnte gezeigt werden, dass Kinder, die lange Zeit vor dem Fernseher bzw. vor einem Bildschirm sitzen häufiger ein Snacking-Verhalten aufweisen, als jene Kinder, die geringere Sitzzeit aufweisen $[2,16,17]$. Daher sollte langandauerndes Sitzen bei Vorschulkindern, Kindern und Jugendlichen reduziert werden.

Schließlich: Bei Kindern unter 6 Jahren nehmen Eltern bzw. alle an der Erziehung beteiligte Erwachsene eine verantwortliche Rolle ein, um ein gesundheitswirksames Bewegungsverhalten im Alltag zu gewährleisten. Diese sollten deshalb von Expertinnen und Experten dabei unterstützt werden, eine altersadäquate bewegungsfördernde Umgebung für Kinder und Jugendliche zu schaffen $[2,18]$.

\section{Zielgruppe}

Die nachfolgenden Bewegungsempfehlungen beziehen sich auf Kinder im Kindergartenalter (3 Jahre bis Schulbeginn) und auf Kinder und Jugendliche ( 6 bis 18 Jahre), unabhängig von Geschlecht, ethnischer Zugehörigkeit und Körper-, Sinnes- oder Mentalbehinderungen.

\section{Empfehlungen für Kinder im Kindergartenalter}

Bewegung soll allen Kindern im Kindergartenalter von den Eltern und betreuenden Erwachsenen täglich ermöglicht werden. Kinder im Kindergartenalter sollen täglich mindestens 3 Stunden körperlich aktiv sein. Diese Zeitspanne kann über den Tag verteilt werden. Von diesen täglichen 3 Stunden Bewegung sollen mindestens 60 Minuten (1 Stunde) für Bewegung mit mittlerer bis höherer Intensität genützt werden.

Auf Freude an der Bewegung, altersentsprechende Bewegungsformen sowie ein möglichst breites motorisches Spektrum soll geachtet werden.

Langandauerndes Sitzen soll vermieden beziehungsweise immer wieder durch Bewegung unterbrochen werden. 


\section{Empfehlungen für Kinder und Jugendliche}

Bewegung soll allen Kindern und Jugendlichen täglich ermöglicht werden.

Kinder und Jugendliche sollen sich täglich mindestens 60

Minuten (1 Stunde) bewegen.

Zur Förderung der Ausdauer soll der Großteil der täglichen mindestens 60-minütigen Bewegung mit mittlerer oder höherer Intensität ausgeübt werden. An 3 Tagen der Woche wird ausdauerorientierte Bewegung mit höherer Intensität empfohlen.

Als Teil der täglichen 60 Minuten Bewegung werden an mindestens 3 Tagen der Woche muskelkräftigende und knochenstärkende Aktivitäten empfohlen.

Auf Freude an der Bewegung, altersentsprechende Bewegungsformen sowie ein möglichst breites motorisches Spektrum soll geachtet werden.Langandauerndes Sitzen soll vermieden beziehungsweise immer wieder durch Bewegung unterbrochen werden.

\section{Erläuterungen}

\section{Aktivitätsstatus}

Weitgehend inaktive Kinder und Jugendliche (4 bis 18 Jahre) sollen dazu motiviert werden, ihren Bewegungsumfang schrittweise zu erhöhen, wobei die Bewegungsformen so zu wählen sind, dass bei der Ausübung Freude erlebt wird und die Verletzungsgefahr gering bleibt. Hierfür eignen sich für Kinder unter 6 Jahren Bewegungsspiele mit Aufgabencharakter wie z. B. ein als Schatzsuche verpackter Orientierungslauf.

Eine Zusammenarbeit mit Bewegungsexpertinnen oder -experten könnte v. a. für inaktive Kinder mit Körper-, Sinnes- oder Mentalbehinderung hilfreich sein, um zu verstehen, welche Aktivitäten und welche Bewegungsumfänge geeignet sind. Bewegungsexpertinnen und -experten können auch die Aufrechterhaltung der Motivation unterstützen.

Bereits bewegungsaktive Kinder und Jugendliche sollen vom sozialen Umfeld motiviert werden, den Bewegungsumfang aufrecht zu erhalten bzw. weiter zu erhöhen. Ziel ist es, diese dabei zu unterstützen, (weitere) Sportarten zu erlernen oder einem Sportverein beizutreten, um an strukturierten Bewegungsangeboten teilzunehmen [19].

Kinder und Jugendliche, die bereits sehr aktiv sind, weil sie z. B. in einem Verein regelmäßig eine Sportart ausüben, sollen bei der Vereinbarkeit mit den schulischen Anforderungen unterstützt werden [19].

\section{Art der Bewegung}

Die Gesundheit durch Bewegung zu stärken und die Bewegungskompetenz durch vielfältige Angebote zu verbessern kann längerfristig gelingen, wenn ein freudvolles Erleben von Bewegung ermöglichet wird. Im Wesentlichen kann dies durch altersentsprechende Bewegungsformen gelingen, die ein breites motorisches Spektrum an Bewegungsaufgaben umfassen.

Geeignete Bewegungsformen für Kinder im Kindergartenalter sind bspw. Herumtollen und Spielen am Spielplatz, kindgerechte
Bewegungsspiele (Spiele, die z. B. Gesang, Geschichten oder Rollenspiele enthalten), Tretroller oder (Lauf-)Rad fahren. Während des „freien Spiels“ im Kindergarten sollen die Bewegungsaufgaben (z. B. balancieren, springen, laufen, klettern, rutschen, tanzen, werfen usw.), die motorischen Beanspruchungsformen (Ausdauer, Kraft und Schnelligkeit) sowie die Bewegungsintensitäten rasch wechseln. Möglicherweise reicht das freie Spielen für Kinder im Kindergartenalter nicht aus, um ein breites Spektrum motorischer Fertigkeiten anzuwenden. Zur Förderung des Bewegungsapparats auf vielfältige Weise eignen sich neben dem freien Spiel daher auch strukturierte und angeleitete Bewegungseinheiten, wie sie z. B. in Sportvereinen oder Bewegungsexpertinnen und -experten angeboten werden $[19,20]$.

Für Kinder in der Primärstufe bzw. in der Volksschule sind Bewegungsspiele mit spielerischer Anwendung motorischer Fähigkeiten sowie das Erlernen motorischer Fertigkeiten wie Radfahren, Schwimmen, Kombinationen motorischer Aufgaben am Boden und an Geräten und Ballspiele und ähnliches mehr geeignet. Während der Pausen oder in der freien Spielzeit sollen Kinder im Volksschulalter die Bewegungsformen (z. B. Springen, Laufen, Klettern, Werfen usw.), die motorischen Beanspruchungsformen (Ausdauer, Kraft und Schnelligkeit) sowie die Bewegungsintensitäten rasch wechseln. Dieses breite motorische Spektrum gehört zum Bewegungsrepertoire von Kindern im Volksschulalter, wenn sie den Bewegungsraum dafür erhalten. Sie benötigen üblicherweise kein angeleitetes Muskeltraining, weil muskelkräftigende Übungen Teil der Bewegungen im Rahmen des freien Spiels sind. Wenn Kinder Freude an muskelkräftigenden Programmen haben, ist das zu begrüßen. Das Training ist nicht gesundheitsschädlich, wenn es richtig und unter Aufsicht durchgeführt wird [1].

Jugendliche erreichen die Bewegungsempfehlungen im Rahmen der aktiven Mobilität (Zufußgehen, Radfahren), des freien Spiels, im schulischen Setting, während der Ausübung von Sportarten und in angeleiteten Kursen. Angeleitete Kurse können auch muskelkräftigende Übungen wie Gewichtheben, Übungen mit elastischen Bändern oder Übungen mit dem eigenen Körpergewicht (z. B. Liegestütz) beinhalten. Muskelkräftigende Übungen tragen dann zur Erfüllung der Bewegungsempfehlungen bei, wenn damit eine mittlere bis hohe Anstrengung verbunden ist und dabei die großen Muskelgruppen wie Bein-, Hüft-, Brust-, Rücken-, Bauch-, Schulterund Armmuskulatur beansprucht werden. Muskulatur kräftigende Bewegung ist gleichzeitig auch knochenstärkende Bewegung, da Aufbau und Masse von Muskeln sehr gut mit dem Aufbau und der Masse von Knochen korrelieren. Weitere Bewegungsformen, die dem Knochenaufbau dienen, sind Aktivitäten, bei denen der Knochen einen Reiz, sich selbst zu stärken, erhält, also sogenannte „High-impact“ Sportarten, die Elemente des Hüpfens, Springens und Laufens beinhalten.

Zur Förderung der psychosozialen Gesundheit und um Verletzungen vorzubeugen, sollten sich Jugendliche eine fundierte Kompetenz für das Krafttraining aneignen oder bei der Ausübung von Expertinnen und Experten angeleitet werden.

Einige Beispiele für gesundheitsförderliche Bewegung für Kinder und Jugendliche (4 bis 18 Jahre) sind in $>$ Tab. 2 dargestellt. Eine Kombination aus allen Bereichen über den Tag verteilt wird empfohlen. 
\Tab. 2 Ausgewählte Beispiele für die Ausdauer verbessernde, die Muskulatur kräftigende und die Knochen stärkende Bewegung für Kinder und Jugendliche.

\begin{tabular}{|c|c|c|}
\hline Wirkrichtung ${ }^{*}$ & Kinder im Kindergarten, 4- bis 6-Jährige & Kinder und Jugendliche, 6- bis 18-Jährige \\
\hline Ausdauer verbessernd & $\begin{array}{l}\text { - Spielen und Bewegung im Freien, Lauf-/Fangspiele auf } \\
\text { kleinerem Raum, Bewegung im Wasser } \\
\text { - Ballspiele mit verschiedenen Ballformen und dabei werfen, } \\
\text { fangen und kicken, Mini-Fußball }\end{array}$ & $\begin{array}{l}\text { - Schnelles Gehen, Bewegung in der Natur, Radfahren, } \\
\text { Ballspiele, Schatzsuche } \\
\text { - Mountainbiking, Sportspiele wie Fußball, Basketball, } \\
\text { Volleyball und Handball; Orientierungslauf; } \\
\text { Langlaufen }\end{array}$ \\
\hline $\begin{array}{l}\text { Muskulatur kräftigend auch } \\
\text { Ausdauer verbessernd }\end{array}$ & $\begin{array}{l}\text { - Dreirad- und Laufradfahren, Balancieren auf einer Linie } \\
\text { oder auf einem Seil am Boden, auf einem Bein stehen } \\
\text { - Klettern, Rangeln, Bewegung im Gelände, Kinderturnen, } \\
\text { - leichtathletische Bewegungsformen, Skifahren, } \\
\text { Langlaufen, an Geräten Turnen, Schwimmen }\end{array}$ & $\begin{array}{l}\text { - Stufensteigen; Kraftübungen mit Eigengewicht und } \\
\text { mit Bändern } \\
\text { - Kraftübungen an Geräten oder mit freien Gewich- } \\
\text { ten, Schwimmen, Turnen } \\
\text { - Klettern, Ski fahren, Snowboarden, Bergsteigen }\end{array}$ \\
\hline Knochen stärkend & $\begin{array}{l}\text { - Hüpfen und Springen, Laufen } \\
\text { - Klettern, Seilhüpfen/-springen } \\
\text { - Kinderturnen }\end{array}$ & $\begin{array}{l}\text { - } \text { Hüpfen und Springen, Seilspringen (Boxtraining) } \\
\text { Handball), mit/Sprungbewegung (z. B. Volleyball, } \\
\text { Basketball), mit Stoppbewegungen (z. B. Tennis), } \\
\text { - Parcours, Ski-Freestyle, Geräteturnen }\end{array}$ \\
\hline
\end{tabular}

\section{Wöchentlicher Bewegungsumfang}

Kinder unter 6 Jahren sollten sich den ganzen Tag über bewegen können, um das körperliche Wachstum und die kognitive Entwicklung sowie die Entwicklung der Sinnesorgane zu fördern.

Basierend auf der verfügbaren Evidenz zur Wirksamkeit von Bewegung im Kleinkindalter (0-5 Jahre) ist zu empfehlen, dass Eltern bereits ihr Neugeborenes über 30 Minuten am Tag verteilt in der Wachzeit in die Bauchlage drehen sollten, um die organische und kognitive Entwicklung zu fördern. Kleinkinder sollten so oft wie möglich am Tag die Möglichkeit bekommen, verschiedene Bewegungsformen beim Spielen auszuprobieren, um sich auf diese Weise ein breites Spektrum an motorischen Fertigkeiten anzueignen $[15,19,21]$. Neben regelmäßiger Bewegung von geringer bis anstrengender Intensität fördern bereits kurzdauernde Bewegungseinheiten kognitive Funktionen wie das Erinnerungsvermögen, die Konzentrationsfähigkeit, die Lerngeschwindigkeit und die Fähigkeit, Worte und Zahlen („literacy“) in diesem Alter zu lernen [19].

Insgesamt sollten sich Kinder ab zwei Jahren täglich mindestens 3 Stunden bewegen können, d. h. um ein gesundes Aufwachsen zu fördern, sollte deren Bewegungsfreiheit nicht eingeschränkt werden. Sitzzeiten vor dem Bildschirm oder am Handy sollten vermieden werden und bei den 2- bis 4-Jährigen allenfalls nicht mehr als 1 Stunde über den Tag verteilt ausmachen. Neugeborene und Kinder $\leq 1$ Jahr sollten überhaupt nicht mit Bildschirmaktivität (Handy, Tablet, TV, usw.) konfrontiert werden [15].

Kinder und Jugendliche im Alter von 6-18 Jahren sollten sich täglich 60 Minuten oder mehr mit mittlerer bis hoher Intensität an allen Tagen in der Woche aerob, d. h. Sauerstoff verbrauchend bewegen. An mindestens 3 Tagen davon sollten sie Muskulatur kräftigende und Knochen stärkende Bewegungsformen durchführen, um einen effektiven Gesundheitsnutzen zu erzielen [15, 19].

Zu beachten ist, dass Jugendliche sich selbständig organisieren, um Sport zu treiben oder sich zu bewegen. Sie trainieren alleine oder in der Gruppe. Während dieser Zeit kann es auch zu einem deutlichen Abfall des Bewegungsumfangs kommen. Aufgrund des dokumentierten Geschlechtsunterschieds in Bezug auf den Bewegungsumfang wird empfohlen, neben speziellen Angeboten für männliche Jugendliche auch und v. a. Mädchen im Jugendalter mit für sie attraktiven Bewegungsangeboten zu fördern [19, 22].

\section{Besondere Hinweise}

\section{Soziale Unterstützung}

Da Kindergartenkinder noch sehr stark auf die Unterstützung und Förderung durch Erwachsene angewiesen sind, müssen Eltern sowie Pädagoginnen und Pädagogen über die Bewegungsempfehlungen aufgeklärt werden und dabei unterstützt werden, eine bewegungsfördernde Umgebung zu schaffen und Bewegung im Alltag altersentsprechend mit den Kindern umzusetzen.

\section{Bewegung für Kinder unter 3 Jahren}

Im Jahr 2019 wurden von der Weltgesundheitsorganisation Bewegungsempfehlungen für Säuglinge (unter 1 Jahr) und Kleinkinder ( 1 bis unter 3 Jahre) veröffentlicht [15]. Da es derzeit jedoch keine gut abgesicherten Daten zur Gesundheitswirksamkeit eines definierten Bewegungsumfanges für diese Zielgruppe gibt, wird diese Altersgruppe noch nicht dezidiert in die Österreichischen Bewegungsempfehlungen aufgenommen.

\section{Inaktivität}

Für Kinder und Jugendliche wird empfohlen, längere Inaktivität, wie langandauerndes Sitzen vor einem Bildschirm, zu vermeiden. Strategien hierfür sind, die Sitzdauer zu reduzieren und die aktive Mobilität sowie Spiele im Freien zu fördern. Für Jugendliche gilt, langandauerndes Sitzverhalten vor dem Bildschirm zu vermeiden und so oft wie möglich zu unterbrechen.

International wird zudem empfohlen, dass Kinder im Kindergartenalter Bildschirmmedien nicht mehr als 1 Stunde über den Tag verteilt nutzen [15]. 


\section{Danksagung}

Die Erstellung der Österreichischen Bewegungsempfehlungen und diese open access Publikation wurden dankenswerter Weise von der Gesundheit Österreich GmbH, Geschäftsbereich Fonds Gesundes Österreich, und der Österreichischen Gesellschaft für Public Health unterstützt.

\section{Interessenskonflikt}

Die Autorinnen/Autoren geben an, dass kein Interessenkonflikt besteht.

\section{Literatur}

[1] Fonds Gesundes Österreich Österreichische Empfehlungen für gesundheitswirksame Bewegung. Wien: Bundesministerium für Gesundheit, Geschäftsbereich Fonds Gesundes Österreich; 2010

[2] U.S. Department of Health and Human Services 2018 Physical Activity Guidelines Advisory Committee Scientific Report. Washington, DC: US Department of Health and Human Services; 2018

[3] Miko HC, Zillmann N, Ring-Dimitriou S et al. Auswirkungen von Bewegung auf die Gesundheit. Gesundheitswesen 2020; 82 (Suppl. 3): S184-S195

[4] The American College of Obstericians and Gynecologists ACOG Committee Opinion: Physical Activity and Exercise During Pregnancy and the Postpartum Period. Obstetrics \& Gynecology 2020; 135: e178-e188

[5] Mottola MF, Davenport MH, Ruchat SM et al. 2019 Canadian guideline for physical activity throughout pregnancy. Br J Sports Med 2018; 52: 1339-1346

[6] Ferrari N, Graf C. Bewegungsempfehlungen für Frauen während und nach der Schwangerschaf. Das Gesundheitswesen 2017; 79: S36-\$39

[7] Korsten-Reck U, Marquardt K, Wurster K. Schwangerschaft und Sport. Deutsche Zeitschrift für Sportmedizin 2009; 60: 117-121

[8] Sulprizio M, Kleinert J. Sport in der Schwangerschaft: Leitfaden für die geburtshilfliche und gynäkologische Beratung. Berlin, Heidelberg: Springer; 2016

[9] Kahlmeier S, Hartmann F, Martin-Diener E et al. Schweizer Bewegungsempfehlungen für schwangere und postnatale Frauen. Schweizer Zeitschrift für Ernährungsmedizin 2018; 4: 14-19
[10] Macfarlane AJ, Blondel B, Mohangoo AD et al. Wide differences in mode of delivery within Europe: risk-stratified analyses of aggregated routine data from the Euro-Peristat study. BJOG 2016; 123: 559-568

[11] Bundeszentrale für gesundheitliche Aufklärung Sonderheft 03: Nationale Empfehlungen für Bewegung und Bewegungsförderung. Köln: BzgA; Im Internet: www.bzga.de/infomaterialien/fachpublikationen/sonderheft-03-nationale-empfehlungen-fuer-bewegung-undbewegungsfoerderung; Stand: 29.04.2020

[12] BASPO BfS, BAG BfG, Schweiz G et al. Gesundheitswirksame Bewegung. Magglingen: BASPO. 2013

[13] Okely AD, Ghersi D, Hesketh KD et al. A collaborative approach to adopting/adapting guidelines - The Australian 24-Hour Movement Guidelines for the Early Years (Birth to 5 years): an integration of physical activity, sedentary behavior, and sleep. BMC Public Health 2017; 17: 869

[14] Pate RR, Hillman CH, Janz KF et al. Physical Activity and Health in Children Younger than 6 Years: A Systematic Review. Med Sci Sports Exerc 2019; 51: 1282-1291

[15] World Health Organization WHO guidelines on physical activity, sedentary behaviour and sleep for children under 5 years of age. Geneva: World Health Organization; 2019

[16] Blundell JE, Gibbons C, Caudwell P et al. Appetite control and energy balance: impact of exercise. Obes Rev 2015; 16(Suppl 1): 67-76

[17] Manios Y, Kourlaba G, Kondaki K et al. Obesity and television watching in preschoolers in Greece: the GENESIS study. Obesity (Silver Spring) 2009; 17: 2047-2053

[18] Ring-Dimitriou S, Freudenthaler T, Aistleitner V et al. SALTO - Study Protocol and Rationale of a Community-Oriented Obesity Prevention Program in the Kindergarten. Obes Facts 2018; 11: 234-246

[19] U.S. Department of Health and Human Services Physical Activity Guidelines for Americans. $2^{\text {nd }}$ edition. Washington, DC: US Department of Health and Human Services; 2018

[20] O'Malley G, Ring-Dimitriou S, Nowicka P et al. Physical Activity and Physical Fitness in Pediatric Obesity: What are the First Steps for Clinicians? Expert Conclusion from the 2016 ECOG Workshop. Int ] Exerc Sci 2017; 10: 487-496

[21] Tremblay MS, Chaput JP, Adamo KB et al. Canadian 24-Hour Movement Guidelines for the Early Years ( $0-4$ years): An Integration of Physical Activity, Sedentary Behaviour, and Sleep. BMC Public Health 2017; 17: 874

[22] Felder-Puig R, Ramelow D, Teutsch F et al. Gesundheit und Gesundheitsverhalten von österreichischen Lehrlingen. Wien: IfGP; 2020 